Received: 2020-03-26

Accepted: 2020-09-24

Published: 2021-03-31

\section{Spinal Cord Stimulator Trial in a Patient on Chronic Warfarin THERAPY}

\author{
Rana Al-Jumah, MD \\ Jamal Hasoon, MD² \\ Ivan Urits, MD² \\ Amnon A Berger, $\mathrm{MD}, \mathrm{PhD}^{2}$ \\ Omar Viswanath, $\mathrm{MD}^{3-5}$ \\ Alaa Abd-Elsayed, MD6 \\ Musa Aner, MD²
}

Background: Spinal cord stimulation (SCS) is a rapidly growing interventional treatment modality in chronic pain. Pain physicians are faced with the decision on how to manage patients on anticoagulation therapy given the risk of epidural hematomas.

Case Report: We describe a patient with a history of atrial fibrillation and prior pulmonary embolism on chronic anticoagulation. The patient was planned to undergo an SCS trial, but was unable to discontinue all anticoagulation during the length of the trial. Utilizing a multidisciplinary approach, the patient discontinued warfarin 5 days prior to the procedure and began a therapeutic dose of low molecular weight heparin (LMWH). The final dose of LMWH was given 24 hours before the trial procedure. The patient then started prophylactic dosing of LMWH 24 hours after the trial procedure and continued that regimen for the course of the SCS trial. The last dose of prophylactic LMWH was given 24 hours before removal of the trial leads and the patient restarted 3 days of therapeutic LMWH along with resuming his normal anticoagulation regimen after lead removal. The patient was able to undergo a successful SCS trial and will be pursuing a SCS implant with further anticoagulation management.

Conclusion: This case demonstrates a possible strategy for managing patients who requiring anticoagulation therapy during the course of their SCS trial phase.

Although a single-electrode array proved to be efficacious, using 2 electrode arrays improves the anatomic coverage of the painful areas and allows for greater optionality in electrode selections to avoid plasticity.

Key words: Spinal cord stimulation, anticoagulation, chronic pain, post laminectomy syndrome

From: ${ }^{1 B}$ Baylor College of Medicine, Department of Anesthesiology, Houston, TX; ${ }^{2}$ Beth Israel Deaconess Medical Center, Department of Anesthesia, Critical Care, and Pain Medicine, Harvard Medical School, Boston, MA; ${ }^{3}$ Valley Anesthesiology and Pain Consultants - Envision Physician Services, Phoenix, AZ; ${ }^{4}$ University of Arizona College of Medicine - Phoenix, Department of Anesthesiology, Phoenix, AZ; ${ }^{5}$ Creighton University School of Medicine, Department of Anesthesiology, Omaha, NE; ${ }^{6}$ Anesthesiology, University of Wisconsin School of Medicine and Public Health, Madison, WI

Corresponding Author: Jamal Hasoon,MD E-mail: Jhasoon@bidmc.harvard.edu Disclaimer: There was no external funding in the preparation of this manuscript.

Conflict of interest: Each author certifies that he or she, or a member of his or her immediate family, has no commercial association (i.e., consultancies, stock ownership, equity interest, patent/licensing arrangements, etc.) that might pose a conflict of interest in connection with the submitted manuscript. 


\section{BACKGROUND}

Interventional pain procedures are a major component of a multimodal treatment regimen in pain medicine. Many patients who suffer from chronic pain are candidates for neuraxial interventional procedures such as spinal cord stimulation (SCS), which is utilized for pain relief. As these procedures become more common, patients with numerous comorbidities, including those requiring anticoagulation, are receiving these treatments. Pain physicians are increasingly faced with the decision of how to manage these comorbidities and their potential for causing complications.

SCS lead placement involves the use of a large-gauge introducer needle. Obtaining epidural access along with lead placement may cause significant trauma in the epidural space leading to hematoma formation (1). The most important risk factor associated with formation of an epidural or spinal hematoma is the use of anticoagulants, either at the time of the interventional procedure or upon epidural catheter removal (2). Recently, the American Society of Regional Anesthesia and Pain Medicine (ASRA) developed updated anticoagulation guidelines and recommendations for interventional pain procedures. A summary of the guidelines for specific anticoagulants is seen in Table 1.

We describe a challenging case of a patient with a history of atrial fibrillation and a prior pulmonary embolism taking warfarin therapy chronically for prophylactic anticoagulation. The patient was scheduled to undergo a SCS trial but needed adjustment on the anticoagulation medication prior to the procedure. We describe our approach to this interesting case.

\section{CASE}

The patient was a 79-year-old man with a past medical history of atrial fibrillation, essential thrombocythemia, pulmonary embolism, hypertension, and postlaminectomy syndrome with chronic lower back pain and radiculopathy. He had an L4-L5 and L5-S1 posterior fusion several years in the past, which did not alleviate his pain. The patient was undergoing a variety of treatments for his chronic pain including multiple rounds of physical therapy, medication management, and several interventional pain procedures to attempt to address his pain. Medication management included nonsteroidal anti-inflammatory drugs (NSAIDs), neuropathic agents, muscle relaxants, and opioids. He also underwent several rounds of epidural steroid injections with minimal relief. The patient continued to endorse severe axial back pain as well as lumbar radiculopathy with a pain score of 10 of 10 in severity. He was ultimately deemed a candidate for SCS pending evaluation and management of his anticoagulation status.

A multidisciplinary plan regarding the patient's anticoagulation status leading up to the spinal cord stimulator trial procedure was made in coordination with the patient's cardiologist, hematologist, pain management physician, and anesthesiologist. The decision was made

Table 1. Recommended guidelines for stopping anticoagulation before interventional procedures.

\begin{tabular}{|l|l|l|l|l|}
\hline \multicolumn{1}{|c|}{ Anticoagulants } & Low-risk Procedures & \multicolumn{1}{|c|}{$\begin{array}{c}\text { Intermediate-risk } \\
\text { Procedures }\end{array}$} & High-risk Procedures & \multicolumn{1}{c|}{ When to Restart } \\
\hline Warfarin & No specific time frame & 5 days, INR normal & 5 days, INR normal & $6 \mathrm{~h}$ after procedure \\
\hline IV Heparin & $6 \mathrm{~h}$ after last dose & $6 \mathrm{~h}$ after last dose & $6 \mathrm{~h}$ after last dose & $2 \mathrm{~h}$ after procedure \\
\hline $\begin{array}{l}\text { Subcutaneous heparin, } \\
\text { BID and TID }\end{array}$ & $6 \mathrm{~h}$ after last dose & $6 \mathrm{~h}$ after last dose & $24 \mathrm{~h}$ after last dose & $\begin{array}{l}2 \mathrm{~h} \text { for low-risk } \\
6-8 \mathrm{~h} \text { for intermediate- and } \\
\text { high-risk }\end{array}$ \\
\hline Enoxaparin therapeutic & $24 \mathrm{~h}$ after last dose & $24 \mathrm{~h}$ after last dose & $24 \mathrm{~h}$ after last dose & $\begin{array}{l}4 \mathrm{~h} \text { for low-risk } \\
12-24 \mathrm{~h} \text { for intermediate- and } \\
\text { high-risk }\end{array}$ \\
\hline Enoxaparin prophylactic & $12 \mathrm{~h}$ after last dose & $12 \mathrm{~h}$ after last dose & $\begin{array}{l}4 \mathrm{~h} \text { for low-risk } \\
12-24 \mathrm{~h} \text { for intermediate- and } \\
\text { high-risk }\end{array}$ \\
\hline Clopidogrel & No specific time frame & 7 days after dose & 7 days after dose & $12-24 \mathrm{~h}$ after procedure \\
\hline Aspirin & No specific time frame & $\begin{array}{l}\text { Procedures that pose } \\
\text { increased risk due to } \\
\text { anatomical location }\end{array}$ & $\begin{array}{l}\text { Primary prophylaxis is } \\
6 \text { days }\end{array}$ & $24 \mathrm{~h}$ after procedure \\
\hline
\end{tabular}

Abbreviations: BID, twice a day; INR, international normalized ratio; IV, intravenous; TID, 3 times a day 
to have the patient discontinue warfarin 5 days prior to the procedure and begin a therapeutic dose of lowmolecular-weight heparin (LMWH). The patient's final dose of LMWH would be given 24 hours before the trial procedure. The patient would then start prophylactic dosing of LMWH 24 hours after the trial procedure and would continue that regimen for the course of the SCS trial. The last dose of prophylactic LMWH would be given 24 hours before removal of the trial leads and the patient would restart 3 days of therapeutic LMWH along with resuming his normal anticoagulation regimen with warfarin after lead removal.

The patient underwent a successful trial using a 10$\mathrm{kHz}$ SCS system. Two trial leads were placed with one at the superior endplate of T8 and the second lead at the superior endplate of T9 (Fig. 1). The patient underwent a 5-day trial and reported $>65 \%$ improvement of his chronic back pain and radiculopathy. When the SCS trial ended, LMWH was held on the day of the lead removal. He reported significant improvement in his daily function from the pain relief and will pursue permanent implantation in the near future with similar planning of his anticoagulation status.

\section{DISCUSSION}

Anticoagulation is typically started on patients with atrial fibrillation, as they have a 5-fold increased risk for thromboembolic events, accounting for approximately $15 \%$ of all strokes in the United States. Our patient additionally had a prior pulmonary embolism after stopping anticoagulation during a previous surgery, which the American College of Cardiology and the American Heart Association consider high risk for further thrombosis and recommend continuation of anticoagulation therapy.

For many, including our patient, warfarin is a common choice for stroke prevention $(3,4)$. Warfarin acts by inhibiting gamma-carboxylation of vitamin K-dependent coagulation factors (II, VII, IX, X) and proteins C and S. The international normalized ratio (INR) is the most common laboratory measurement to assess the coagulation status of patients taking warfarin (5). According to the ASRA guidelines, warfarin should be stopped at least 5 days before any high-risk procedure, including a SCS implant and trial. Those patients should also have a normalized INR of 1.2 or less. Recommendations for patients on warfarin receiving interventional spine and pain procedures are summarized in Table 2 .

Since this patient had a complicated medical history, including a previous pulmonary embolism, bridge

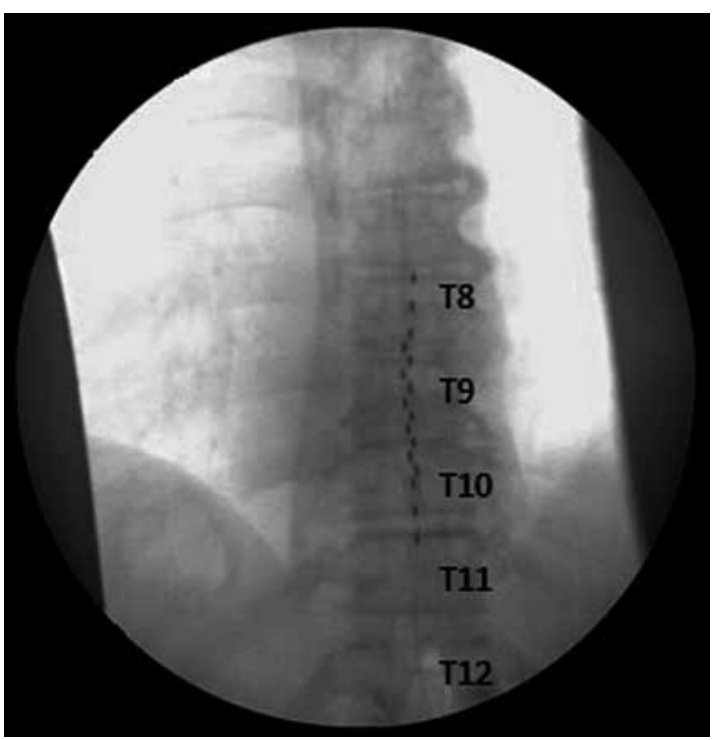

Fig. 1. Anteroposterior radiographs of the intraoperative imaging demonstrating successful lead placement towards the T8 and T9 vertebrae.

Table 2. Warfarin recommendations for interventional pain procedures.

Warfarin Recommendations for Interventional Pain Procedures

- Low-risk procedures may be safe with INR less than 3 .

- Warfarin should be stopped 5 days prior to high- or intermediaterisk procedures

- INR should be less than or equal to 1.2 for high- or intermediaterisk procedures

- If patient is at high risk for venous thrombosis, then bridge therapy with LMWH may be utilized.

Abbreviations: LMWH, low-molecular-weight heparin; INR, international normalized ratio

therapy with LMWH was utilized to prevent further thromboembolic events (6). The ASRA guidelines state that bridging therapy with a short-acting anticoagulant like LMWH minimizes the risk of bleeding during the procedure $(1,5)$. LMWH binds to antithrombin to form a complex that irreversibly inactivates factor Xa. Antifactor Xa activity can be measured to assess anticoagulation status $(1,5)$. Traditionally, patients undergoing a SCS implant and trial have LMWH discontinued 12 hours (if given prophylactic dose) or 24 hours (if given therapeutic dose) prior to the procedure. Further recommendations regarding $\mathrm{LMWH}$ are summarized in Table 3.

\section{CONCLUSIONS}

This interesting case details the unique challenges of managing anticoagulation for spinal cord stimula- 
Table 3. LMWH recommendations for interventional pain procedures.

Low-Molecular-Weight Heparin Recommendations for Interventional Pain Procedures

- Caution should be taken when using NSAIDS, SSRIs, or other antiplatelet and anticoagulants concomitantly with LMWH

- Discontinue LMWH 24 hours prior to intermediate- or high-risk procedures if dose given was therapeutic

- Discontinue LMWH 12 hours prior to intermediate- or high-risk procedures if dose given was prophylactic

- LMWH resumed 4 hours after low-risk procedure

- LMWH resumed 12-24 hours after intermediate- or high-risk procedures

Abbreviations: LMWH, low-molecular-weight heparin; NSAIDS, nonsteroida anti-inflammatory drug; SSRI, selective serotonin reuptake inhibitors

\section{REFERENCES}

1. Narouze S, Benzon HT, Provenzano DA, et al. Interventional spine and pain procedures in patients on antiplatelet and anticoagulant medications: Guidelines from the American Society of Regional Anesthesia and Pain Medicine, the European Society of Regional Anaesthesia and Pain Therapy, the American Academy of Pain Medicine, the International Neuromodulation Society, the North American Neuromodulation Society, and the World Institute of Pain. Reg Anesth Pain Med 2015; 40:182-212.

2. Vandermeulen EP, Van Aken $\mathrm{H}$, Vermylen J. Anticoagulants and spinal-epidural anesthesia. Anesth Analg 1994; 79:1165-1177.

3. The Boston Area Anticoagulation Trial for Atrial Fibrillation Investigators. The effect of low-dose warfarin on the risk of stroke in patients with nonrheumatic atrial fibrillation. N Engl J Med 1990; 323:1505-1511.

4. Reiffel JA. Atrial fibrillation and stroke: Epidemiology. Am J tor lead placement. Our patient requires long-term anticoagulation therapy, and with the help of a multidisciplinary approach, was able to undergo a SCS trial without any complications. Our patient is planning to undergo permanent SCS implantation in the future and we look forward to following this patient's progress and reporting on his results as they become available. This case provides a possible strategy for managing patients who require anticoagulation therapy during the course of their SCS trial phase.

Med 2014; 127:15-16

5. Narouze S, Benzon HT, Provenzano D, et al. Interventional spine and pain procedures in patients on antiplatelet and anticoagulant medications (second edition): Guidelines from the American Society of Regional Anesthesia and Pain Medicine, the European Society of Regional Anaesthesia and Pain Therapy, the American Academy of Pain Medicine, the International Neuromodulation Society, the North American Neuromodulation Society, and the World Institute of Pain. Reg Anesth Pain Med 2018; 43:225-262.

6. January CT, Wann LS, Calkins H, et al. 2019 AHAVACC/HRS focused update of the 2014 AHA/ACC/HRS guideline for the management of patients with atrial fibrillation: A report of the American College of Cardiology/American Heart Association Task Force on Clinical Practice Guidelines and the Heart Rhythm Society. Circulation 2019; 74:104-132. 